\title{
Zur Hönl-Dehnenschen Formulierung des Machschen Prinzips
}

\author{
J. EHLERS und E. SCHÜCKING * \\ Department of Physics, The University of Texas, Austin, USA
}

Eingegangen am 27. Mai $1967 \star \star$

It is shown that the classification of curved space-times into Machian and antiMachian world models proposed by HöNL and DEHNEN is inadequate. We prove that even the static Einstein model would be anti-Machian according to the definition of these authors, in contrast to their assertion. The proof is based on the theorem, established in this paper, that any Robertson-Walker space-time admits regular sourcefree Maxwell fields.

In einer Reihe von Arbeiten ${ }^{1}$ haben HöNL und DEHNEN eine neue Fassung des Machschen Prinzips in der Einsteinschen Gravitationstheorie vorgeschlagen und behauptet, daß alle räumlich geschlossenen Weltmodelle diesem Prinzip genügten. Wir werden zeigen, daß die neue Version dieses Prinzips physikalisch unhaltbar ist. Unabhängig davon werden wir die mathematische Behauptung, räumlich geschlossene Weltmodelle seien (im neuen Sinne) Machisch, durch Angabe von Gegenbeispielen widerlegen. Die Gegenbeispiele werden so gewonnen, daß für die Robertson-Walker-Modelle reguläre, quellenfreie Maxwellfelder konstruiert werden.

\section{Kritik der Hönl-Dehnenschen Dynamik}

HöNL und DEHNEN führen ein zeitartiges Einheitsvektorfeld $u^{\mu}$,

$$
u_{\mu} u^{\mu}=1 \text {, }
$$

ein, das Beobachterfeld ${ }^{\star \star \star}$, und zerlegen physikalische Größen bezüglich $u^{\mu}$ in räumliche, zeitliche und eventuell gemischte Anteile. Die

${ }^{1}$ Hönt, H., u. H. Dehnen: Ann. Physik 14, 271 (1964); - Z. Physik 191, 313 (1966), sowie weitere Arbeiten, die in den genannten Schriften zitiert sind.

* Supported by AF-AFOSR grant 45465 and by the Aerospace Research Laboratories, Office of Aerospace Research, US Air Force.

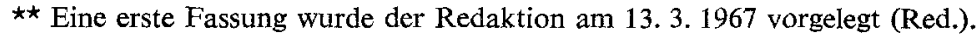

$\star \star \star$ Nicht alle Raumzeit-Mannigfaltigkeiten sind zeitorientierbar, d.h. gestatten global ein stetiges, zeitartiges Einheitsvektorfeld. Eine nicht zeitorientierbare Mannigfaltigkeit kann wie folgt gewonnen werden: Man identifiziere im Minkowskiraum $M$ mit Inertialkoordinaten $t, x_{i}(i=1,2,3)$ alle Punkte, die durch die Abbildungen der 
4-Geschwindigkeit $v^{\mu}$ eines Probekörpers z. B. wird aufgespalten in den zu $u^{\mu}$ orthogonalen Anteil $v^{\mu}$ und den ,Rest“ $v u^{\mu}$,

$$
v^{\mu}=v^{\underline{\mu}}+v u^{\mu}, \quad u_{\mu} v^{\underline{u}}=0, \quad v=u_{\mu} v^{\mu} .
$$

$v$ ist der Lorentzfaktor des Teilchens bezüglich des Beobachters, $m_{0} v$ vein Relativimpuls. Mit der Beobachterzeit $T=\int u_{\mu} d x^{\mu}$ als Parameter kann man dann die Geodätengleichung

$$
\frac{D v^{\mu}}{d T}=0
$$

( $D=$ absolutes Differential längs der Probekörper-Weltlinie) umschreiben in

$$
\frac{D}{d T}\left(m \dot{x}_{\underline{\mu}}\right)=-u_{\mu} \frac{d m}{d T}-m u_{\mu \| \lambda} \dot{x}^{\lambda} \quad\left(m=m_{0} v, \dot{x}^{\mu}=\frac{d x^{\mu}}{d T}\right) .
$$

Raumprojektion bezüglich $u^{\mu}$ gibt

$$
\frac{D_{\perp}}{d T}\left(m \dot{x}_{\underline{\mu}}\right)=-m\left(u_{\underline{\mu \| \lambda}} \dot{x}^{\hat{\lambda}}+\dot{u}_{\mu}\right),
$$

worin $D_{\perp} / d T$ die Raumprojektion der absoluten Ableitung und $\dot{u}_{\mu}$ die 4-Beschleunigung bezeichnen.

Die Zeitableitung $D_{\perp} / d T$ bezieht sich auf (bezüglich $u^{\mu}$ ) räumliche Koordinatenachsen, die längs der Teilchenweltlinie ,,so parallel wie möglich" sind. ( $D_{\perp} / d T$ ist eine Verallgemeinerung der Fermiableitung.) Wenn man statt dessen Achsen verwenden will, die gegenüber den eben genannten mit der lokalen Winkelgeschwindigkeit $\omega_{\lambda \mu}$ des Beobachter-

eigentlich diskreten, fixpunktfreien Isometriegruppe

$$
G:\left\{\begin{array}{l}
x_{i}^{\prime}=x_{i}+n_{i}, \quad n_{i} \text { ganz } \\
t^{\prime}=(-1)^{n_{1} t}
\end{array}\right.
$$

auseinander hervorgehen. Punktidentifikation mittels $G$ erzeugt somit eine CliffordKleinsche Raumform $K$ für die Minkowskiwelt. Ein zeitartiger Vektor $U$ im Nullpunkt von $M$ möge eine positive Zeitkomponente haben. Dann hat der Vektor $W$ im Punkte $t=x_{2}=x_{3}=0, x_{1}=1$, der bezüglich $G$ mit $U$ äquivalent ist, eine negative Zeitkomponente. Ein stetiges, $G$-invariantes Vektorfeld, das in $(0,0,0,0)$ den Wert $U$ hat, muß3 also in mindestens einem Punkt der $x_{1}$-Achse eine verschwindende Zeitkomponente haben. Es gibt daher kein stetiges, nichtverschwindendes, zeitartiges Vektorfeld auf der Mannigfaltigkeit $K$.

Mannigfaltigkeiten wie die soeben konstruierte fallen nicht unter die Hönl-Dehnensche Klassifikation von Lösungen in Machsche und anti-Machsche. Wir danken A. Trautman für eine Diskussion.

Für die Diskussion der Raumformen und der zugehörigen Identifizierungsgruppen, siehe KLEIN, F.: Vorlesungen über Nichteuklidische Geometrie, Berlin 1926. 
feldes rotieren, muß man wie in der Mechanik starrer Körper den Operator $D_{\perp} / d T$ ersetzen durch

$$
\frac{D^{\prime}}{d T}()_{\mu}=\frac{D_{\perp}}{d T}()_{\mu}+()_{\lambda} \omega_{\mu}^{\lambda}, \quad \omega_{\lambda \mu}:=u_{[\lambda, \mu]} .
$$

Dann erhält man statt (5) die Gleichung

$$
\frac{D^{\prime}}{d T}\left(m \dot{x}_{\underline{\mu}}\right)=-m\left(2 \omega_{\mu \lambda} \dot{x}^{\lambda}+q_{\mu \lambda} \dot{x}^{\lambda}+\dot{u}_{\mu}\right),
$$

in der $q_{\mu \lambda}:=u_{(\mu \| \lambda)}$ die Deformationsgeschwindigkeit des Beobachterfeldes bedeutet.

Eine dritte, quasi-Newtonsche Form der Geodätengleichung ist

$$
\begin{aligned}
\frac{D^{\prime \prime}}{d T}\left(m \dot{x}_{\underline{\mu}}\right): & =\frac{D_{\perp}}{d T}\left(m \dot{x}_{\underline{\mu}}\right)+m u_{\underline{\lambda \| \mu}} \dot{x}^{\lambda} \\
& =\left(m \dot{x}_{\underline{\mu}}\right)_{\| \lambda} \dot{x}^{\lambda}+£_{\mu}\left(m \dot{x}_{\underline{\mu}}\right)=m\left(2 \dot{x}^{\lambda} \omega_{\lambda \mu}-\dot{u}_{\mu}\right) .
\end{aligned}
$$

(Der Ausdruck vor dem letzten Gleichheitszeichen ist so zu bilden, daß der zunächst nur längs der Weltlinie des Probekörpers definierte Vektor $m \dot{x}_{\underline{\mu}}$ irgendwie in eine vierdimensionale Umgebung dieser Linie fortzusetzen ist; $f_{u}$ bedeutet die Lie-Ableitung bezüglich $u^{\mu}$.)

Bei einem starren Beobachterfeld, $q_{\lambda \mu}=0$, sind (7) und (8) identisch, und man kann in Analogie zur Newtonschen Mechanik (formuliert mit Hilfe beliebig beschleunigter, starrer Bezugssysteme) die rechte Seite der Gl. (8) als Kraft und die Ausdrücke $2 \omega_{\lambda \mu}=: B_{\mu \lambda}$ und $-\dot{u}_{\mu}=: E_{\mu}$ als Coriolis- und Gravitationsfeldstärke bezeichnen.

Bei einem nichtstarren Beobachterfeld $u^{\mu}$ scheint es uns aber eine unmotivierte, willkürliche Festsetzung zu sein, wenn man wie HöNL und DeHNEN die rechte Seite von (8) als Kraft relativ zu $u^{\mu}$ bezeichnet. Warum z.B. nimmt man nicht die rechte Seite von (7)?

Als Kriterien für eine zweckmäßige Kraftdefinition könnte man etwa folgende Forderungen nehmen:

A) Die ,Zeitableitung“ $\bar{D} / d T$ in der Bewegungsgleichung soll eine metrische Derivation sein, d. h. eine Derivation, die zu $u^{\mu}$ senkrechte Vektoren $w^{\mu}$ in ebensolche Vektoren überführt und die so beschaffen ist, daß aus $(\bar{D} / d T) w^{\mu}=0$ folgt $w_{\mu} w^{\mu}=$ const. (Die „Konstanz" eines Vektors soll die Konstanz seines Betrages implizieren.)

B) Die Zeitableitung der in (4) definierten Relativenergie $m$ soll gleich dem Skalarprodukt aus der Kraft und der Relativgeschwindigkeit $\dot{x}^{\prime} \underline{u}$ sein.

Man überzeugt sich leicht davon, daß die Bewegungsgleichung (7) beide Forderungen erfüllt, (8) hingegen beide Forderungen verletzt. Das hat z.B. die merkwürdige Konsequenz, daß in einem Friedman-Modell 
mit $u^{\mu}$ als 4-Geschwindigkeit des Substrates die Hönl-Dehnensche Kraft auf einen Probekörper zwar verschwindet, der Betrag des Relativimpulses und die Relativenergie aber ständig abnehmen. [Nähme man hingegen G1. (7) als Bewegungsgleichung, so erhielte man eine Kraft, welche diese Abnahme von Impuls und Energie ,erklären“ würde.]

Aus den angeführten Gründen scheint uns die Bezeichnung der in (8) rechts auftretenden Größen als Feldstärken des Gravitations- und Coriolisfeldes (außer in dem sehr speziellen Fall starrer Beobachterfelder, deren Existenz in den meisten nichtstationären Raumzeiten sehr zweifelhaft ist) unangemessen. Wir haben die andere Möglichkeit (7) hier nur angeführt, um die Willkür der Hönl-Dehnenschen Kraftdefinition zu erläutern, nicht um unsererseits eine Kraftdefinition vorzuschlagen. Wir meinen vielmehr, daß EINSTEIN und WeYL überzeugend dargelegt haben, daß wegen der Äquivalenz von (passiver) schwerer Masse und träger Masse die Gravitation in der Bewegungsgleichung nicht auf die Seite der Kraft, sondern auf die der Trägheit gehört. Wenn man dieser fundamentalen Änderung des Begriffssystems der Dynamik nicht folgen will, besteht wohl auch kaum ein Grund, eine Riemannsche Metrik und die Geodätenhypothese einzuführen.

\section{Kritik der Hönl-Dehnenschen Feldgleichungen}

Nach Einführung ihrer Feldstärken setzen HöNL und DeHNEN

$$
F_{\mu v}=u_{v \mid \mu}-u_{\mu \mid v},
$$

so daß $E_{\mu}$ und $B_{\mu \nu}$ mit $F_{\mu \nu}$ so zusammenhängen, wie in der Elektrodynamik. Natürlich ist dann

$$
F_{[\mu \nu, \lambda]}=0 .
$$

Dann wird (dies gilt für die ältere Fassung der Theorie; für die spätere Fassung siehe unsere Bemerkungen am Schluß von Abschnitt 4)

$$
F^{\mu \nu}{ }_{\| v}=\frac{\kappa}{2}\left(j^{\mu}+s^{\mu}\right), \quad j^{\mu}=T^{\mu v} u_{v}
$$

gesetzt und $s^{\mu}$ aus der Einsteinschen Gravitationsfeldgleichung berechnet. Natürlich ist infolge der Definition (11) von $s^{\mu}$

$$
\left(j^{\mu}+s^{\mu}\right)_{\| \mu}=0,
$$

und diese Gleichung wird als Ausdruck der Erhaltung der Energie von Materie $\left(j^{\mu}\right)$ und Gravitationsfeld $\left(s^{\mu}\right)$ gedeutet. Gegen diese Deutung spricht, daß die Identität (12) mit den Gravitationsgleichungen nichts zu tun hat. Es handelt sich in (12) nicht um ein erstes Integral der Feldgleichungen, aus dem man irgendwelche Folgerungen über den Verlauf der Lösungen ziehen könnte aufgrund einer Kenntnis der Cauchydaten, 
sondern nur um eine kinematische Identität wie etwa $\operatorname{div} \omega=0$ in $\operatorname{der}$ klassischen Hydrodynamik.

So wie $\operatorname{div} \omega=0$ daraus folgt, daß $\omega$ als $\frac{1}{2}$ rot $v$ definiert ist, folgt (12) daraus, daß $j^{\mu}+s^{\mu}$ durch die erste Gl. (11) definiert ist. Die Einsteinsche Feldgleichung wird von HöNL und DEHNEN nur dazu benutzt, um $s^{\mu}$ durch $u^{\mu}, g_{\lambda_{\mu}}$ und deren Ableitungen auszudrücken.

Die Analogie zwischen (10), (11) und den Maxwellschen Gleichungen ist formal und durch die Definitionen der Hilfsgrößen $F_{\mu v}, s^{\mu}$ erzwungen. Von einer Ableitung dieser Gleichungen aus den Einsteinschen Feldgleichungen kann man nicht sprechen. [Als Hilfsformeln der relativistischen Hydrodynamik sind übrigens (10) und (11) wohlbekannt; s. z. B. ${ }^{2}$ o)

\section{Das neue Machsche Prinzip}

Eine Lösung $\left(g_{\mu \nu}, T^{\mu v}\right)$ der Einsteinschen Feldgleichungen genügt nach HöNL und DEHNEN genau dann dem (neuen) Machschen Prinzip, wenn für jedes (globale) Beobachterfeld $u^{\mu}$ die Gln. (10) und (11) bei bekannten, aus $u^{\mu}$ berechneten Quellen genau eine reguläre Lösung $F_{\mu \nu}$ haben. [Die Autoren äußern sich nicht ganz klar darüber, ob $F_{\mu \nu}$ auch ein Potential gemäß (9) mit (1) als Nebenbedingung haben muß. Wir werden im folgenden die Gegenbeispiele so konstruieren, daß sie auch noch der Nebenbedingung genügen.]

Zur vorangehenden Kritik möchten wir noch hinzufügen, daß es uns unsinnig erscheint, die Eindeutigkeit von $F_{\mu \nu}$ als eine Bestimmtheit des Führungsfeldes durch die Energie-Impulsverteilung zu interpretieren. Zur Berechnung der Quellgröße $j^{\mu}+s^{\mu}$ muß man nämlich neben $u^{\mu}$ und $T^{\mu v}$ noch die Metrik $g_{\mu v}$ heranziehen. Wenn man jedoch die Metrik kennt, kennt man ja auch die Geodätischen, d. h. das Führungsfeld. Mit anderen Worten: Ein Führungsfeld $\left(F_{\mu \nu}\right)$ wird aus Quellgrößen berechnet, deren Berechnung bereits die Kenntnis der $g_{\mu \nu}$ und damit des Führungsfeldes voraussetzt.

Abgesehen von der Frage der physikalischen Deutung erhebt sich die mathematische Frage, welche Lösungen der Einsteinschen Feldgleichungen Machisch im oben erörterten Sinne sind. Offenbar ist eine Raumzeit anti-Machisch, wenn es in ihr mindestens zwei verschiedene Beobachterfelder $u^{\mu}$ und $v^{\mu}$ so gibt, daß für $H_{\mu v}=\left(v_{v}-u_{v}\right)_{[\mu}-\left(v_{\mu}-u_{\mu}\right)_{\mid v}$ die Gleichung $H^{\mu \nu}{ }_{\| v}=0$ gilt. Dann kann man zu dem zu $u_{\mu}$ gehörenden $F_{\mu \nu}$ das Feld $H_{\mu \nu}$ addieren, ohne (10) oder (11) zu verletzen, und das neue Feld hat auch wieder ein normiertes 4-Potential, nämlich $v_{\mu}$.

Wir werden im nächsten Abschnitt zeigen, daß alle RobertsonWalker-Mannigfaltigkeiten mit einfach zusammenhängenden Raumschnitten anti-Machisch sind, entgegen der Behauptung von HöNL und DEHNEN, wonach diejenigen mit sphärischem Raum Machisch sind.

\footnotetext{
${ }^{2}$ EHLERS, J.: Abhandl. Akad. Wiss. Mainz, Math.-Naturw. K1., Nr. 11, 1961.
} 


\section{Maxwellfelder in Robertson-Walker-Modellen}

Sei $V_{4}=V_{1} \times V_{3}$ eine Produktmannigfaltigkeit mit der Metrik

$$
d s^{2}=d t^{2}-d L^{2}, \quad d L^{2}=\gamma_{i k}\left(x^{l}\right) d x^{i} d x^{k} \quad(i, k, l=1,2,3) .
$$

Dann rechnet man leicht nach, daß

$$
F_{\mu v}\left(x^{l}, t\right)=M_{\mu v}\left(x^{l}\right) e^{-i \omega t}
$$

für $\omega \neq 0$ genau dann die Maxwellgleichungen $F_{[\mu v \mid \lambda]}=0, F^{\mu v}{ }_{\| \nu}=0$ in $V_{4}$ erfüllt, wenn mit der Bezeichnung

die Gleichungen

$$
M_{k 0}=M_{k}
$$

und

$$
i \omega M_{k l}=M_{l \mid k}-M_{k \mid l}
$$

$$
M_{\| l}^{l}=0, \quad-M_{k}^{l \| k}=\left(\omega^{2} \delta_{k}^{l}+R_{k}^{l}\right) M^{k}
$$

(in $V_{3}$ bezüglich der Metrik $d L^{2} ; R_{k}^{l}$ sei der Riccitensor von $d L^{2}$ ) gelten.

Ein Killingvektor $\xi^{l}$ in $V_{3}$ erfüllt infolge der Integrabilitätsbedingung $£_{\xi} \Gamma_{k l}^{j}=0$ die Beziehung

$$
\xi^{l|| k}=R_{k}^{l} \xi^{k},
$$

so daß sich für einen Killingvektor $M^{k}$ die Gln. (17) auf die Eigenvektorgleichung

$$
R_{k}^{l} \xi^{k}=-\frac{\omega^{2}}{2} \xi^{l}
$$

reduzieren. Es gilt also das folgende

Lemma 1: Wenn $\xi^{l}$ ein Killingvektor und zugleich ein Ricci-Eigenvektor zum Eigenwert $-\omega^{2} / 2 \neq 0$ der Riemannschen Mannigfaltigkeit $V_{3}$ ist, dann ist (14) mit

$$
M_{k 0}=\xi_{k}, \quad M_{k l}=\frac{1}{i \omega}\left(\xi_{l \mid k}-\xi_{k \mid l}\right)=\frac{2 i}{\omega} \xi_{k \| l}
$$

eine Lösung der homogenen Maxwellgleichungen in der Produktmannigfaltigkeit $V_{4}$ mit der Metrik (13).

Wenn $V_{3}$ insbesondere ein Raum konstanter Krümmung $K$ (=Gaußsches Krümmungsmaß) ist, ist wegen $R_{k}^{l}=-2 K \delta_{k}^{l}$ für jeden Killingvektor die G1. (19) erfüllt mit $\omega=2 \sqrt{K}$. Da die einfach zusammenhängenden Raumformen globale sechsdimensionale Bewegungsgruppen zulassen, sind ihre Killingvektoren überall regulär. Wir erhalten

Lemma 2: Wenn $\xi^{l}$ ein Killingvektor im Raum $V_{3}$ konstanter Krümmung $K \neq 0$ ist, dann erfüllt der nach (20) und (14) mit $\omega=2 \sqrt{K}$ gebildete Bivektor $F_{\mu \nu}$ die homogenen Maxwellgleichungen in der Produktmannigfaltigkeit $V_{4}$ mit der Metrik (13). Wenn $V_{3}$ einfach zusammen- 
hängt, existieren (global) sechs linear unabhängige, reguläre derartige Maxwellfelder.

Eine Robertson-Walker-Metrik ist wegen

$$
d s^{2}=d t^{2}-a^{2}(t) d L^{2}=a^{2}(t)\left[\left(\frac{d t}{a(t)}\right)^{2}-d L^{2}\right]
$$

im Bereich $a(t)>0$ global konform zu einer Produktmetrik, in der $d L^{2}$ konstante Krümmung \pm 1 oder 0 hat. Die homogenen Maxwellschen Gleichungen sind konform invariant, wenn $F_{\mu v}$ als von der Metrik unabhängiger Tensor aufgefaßt wird. Deshalb folgt aus Lemma 2 der

Satz: Wenn $\xi^{l}$ ein Killingvektor im Raum $V_{3}$ konstanter, nichtverschwindender Krümmung $k= \pm 1$ ist, erfüllt der Bivektor

$$
\begin{gathered}
F_{\mu \nu}=M_{\mu \nu} e^{-2 i \sqrt{k} \vec{t}}, \\
M_{j 0}=\frac{1}{a(t)} \xi_{j}, \quad M_{j l}=\frac{i}{\sqrt{k}} \xi_{j \| l}, \quad \bar{t}=\int \frac{d t}{a(t)}
\end{gathered}
$$

[Komponenten bezüglich $\left(x^{l}, t\right)$, nicht bezüglich $\left(x^{l}, \bar{t}\right)$ ] die homogenen Maxwellgleichungen in der Robertson-Walker-Mannigfaltigkeit $V_{4}$ mit der Metrik (21).

Zusatz: Für verschwindende Krümmung des Raumteils ist $V_{4}$ für $a(t)>0$,räumlich global" konform flach, und jedes Maxwellfeld im Minkowskiraum (z.B. ebene Welle) ist auch eines von $V_{4}$.

Wir haben damit gezeigt, daß alle Friedmanschen Modelle Scharen von regulären, quellenfreien Maxwellfeldern gestatten.

Um den anti-Machschen Charakter (im Sinne von HöNL und DEHNEN) der genannten Modelle vollständig nachzuweisen müssen wir noch zeigen, daß die oben konstruierten Maxwellfelder $F_{\mu v}$ ein Potential der Form $A_{\mu}=v_{\mu}-u_{\mu}$ mit zwei normierten, zeitartigen Vektorfeldern $v_{\mu}, u_{\mu}$ haben. Das ist leicht. Die obigen Felder haben nämlich das raumartige Vektorpotential

$$
A_{\mu}=\left(0, \frac{1}{2 i \sqrt{k}} \xi_{l} e^{-2 i \sqrt{k} \bar{t}}\right)
$$

(wovon für ein reelles Feld natürlich der Realteil zu nehmen ist), und man braucht nur - mit Koordinaten wie in (21) -

$$
u_{0}=\left(1-g^{k l} u_{k} u_{l}\right)^{\frac{1}{2}}, \quad u_{k}=-\frac{1}{2} A_{k}
$$

zu nehmen, dann sind $u_{\mu}$ und $v_{\mu}=A_{\mu}+u_{\mu}$ beide normiert, zeitartig und regulär.

Abschließend möchten wir noch kurz auf die letzte Version des Machschen Prinzips hinweisen, die von HöNL und DEHNEN im Jahre 1966 
vorgeschlagen wurde ${ }^{1}$. Die Feldgleichungen bezüglich eines Beobachterfeldes werden nun in der Form

$$
\begin{gathered}
F_{[\lambda \mu \mid v]}=0, \quad G_{\lambda{ }_{\| \mu}=j_{\lambda}+t_{\lambda},} \\
G_{\mu \nu} u^{\nu}=\frac{2}{\kappa} F_{\mu \nu} u^{\nu}, \quad G_{[\mu \nu} u_{\lambda]}=\frac{1}{2 \kappa} F_{[\mu \nu} u_{\lambda]}
\end{gathered}
$$

geschrieben nach dem Muster der makroskopischen Elektrodynamik. Man verlangt dann als neuestes Mach-Prinzip, daß bei gegebenem $u^{\mu}$ die Gln. (23), (24) bezüglich $F_{\lambda \mu}, G_{\lambda \mu}$ eindeutig lösbar sein sollen. Diese Eindeutigkeit besteht aber wiederum nicht beim Einstein-Kosmos und bei den Friedman-Modellen, wie man durch folgende Überlegung einsehen kann. Die zwei Gln. (24) sind ${ }^{3}$ damit gleichwertig, daß

$$
G^{\lambda \mu}=\frac{1}{2 \kappa} \bar{g}^{\lambda \rho} \bar{g}^{\mu \sigma} F_{\rho \sigma},
$$

gilt, worin

$$
\bar{g}_{\lambda \mu}:=g_{\lambda \mu}-\frac{3}{4} u_{\lambda} u_{\mu}, \quad \bar{g}^{\lambda \mu} \bar{g}_{\mu \nu}=\delta_{v}^{\lambda}
$$

eine Hilfsmetrik ist. Weil $\operatorname{det}\left(\bar{g}_{\lambda \mu}\right)=\frac{1}{4} \operatorname{det}\left(g_{\lambda \mu}\right)$ ist, kann also (23) in

$$
F_{[\lambda \mu, v]}=0, \quad \frac{1}{2 \kappa}\left(\bar{g}^{\lambda \rho} \bar{g}^{\mu \sigma} F_{\rho \sigma}\right)_{; \mu}=j^{\lambda}+t^{\lambda}
$$

umgeschrieben werden, wo „;;“ sich auf die Hilfsmetrik bezieht. Da mit $g_{\lambda_{\mu}}$ auch $\bar{g}_{\lambda \mu}$ eine Robertson-Walker-Metrik ist, wenn für $u^{\mu}$ die 4-Geschwindigkeit des Substrates genommen wird, ist der Nachweis der Nichteindeutigkeit der Lösungen von (23), (24) somit auf den oben von uns behandelten Fall zurückgeführt.

Wir danken Herrn Professor HönL und Herrn Dr. Dehnen für kritische Bemerkungen zu den in dieser Arbeit behandelten Fragen.

Zusatz bei der Korrektur. Im Hinblick auf die auf unsere Arbeit folgende Erwiderung der Herren HöNL und DEHNEN* möchten wir folgende Bemerkungen machen:

1. Die Interpretation der Einsteinschen Gravitationstheorie liegt u.E. durchaus fest. Es ist klar, wie die tatsächlich beobachtbaren Größen wie z.B. Zeiten, Winkel und Lichtintensitäten mathematisch zu repräsentieren sind. Wir sehen nicht, daß HÖNL und DEHNEN etwas zur Interpretation der Theorie beigetragen haben; sie haben weder wirkliche Messungen noch Gedankenexperimente diskutiert. Solange nicht gesagt wird, wie die Größe $t_{\lambda}$ [s. (HD 2.5)] gemessen werden soll, scheint uns für die Interpretation der Theorie nichts gewonnen zu sein, ob man dieser Größe nun den Namen ,Energie-Impulsdichte des Gravitationsfeldes" gibt oder nicht.

2. Man kann die Aussage, daß ein frei fallender Probekörper eine geodätische Weltlinie hat, umformen in die GIn. (7) und (8) (und viele andere!) und damit Gravitationsfeldstärken definieren. Gegen die Einführung solcher Größen als Hilfsgrößen ist

* Diese Zeitschrift, S. 492, im folgenden als HD zitiert.

${ }^{3}$ Gordon, W.: Ann. Physik 72, 421 (1923). 
natürlich nichts einzuwenden. Eine grundlegende Bedeutung kann man ihnen aber nach unserer Meinung nicht beimessen; denn erstens kann man mit Hilfe dieser Feldstärken allein - ohne Kenntnis der Metrik - die Bewegung von Probekörpern nicht berechnen, während diese Feldstärken bei Kenntnis der Metrik zur Berechnung der Probekörperweltlinien nicht nötig sind; und zweitens kann man die grundlegenden Feldgleichungen, nämlich ErNsterns Gleichungen, nicht durch äquivalente Gleichungen für diese Feldstärken allein ersetzen.

3. Die sog. Feldgleichungen (HD 2.3) und der Erhaltungssatz (HD 2.7) haben in folgendem Sinne nichts mit EINSTEINs Feldgleichungen zu tun: Bei gegebener Metrik und gegebenem Beobachterfeld wird $F_{\lambda \mu}$ durch (9) oder (HD 2.1), $G_{\lambda \mu}$ durch (HD 2.2) und daraufhin $j_{\lambda}+t_{\lambda}$ durch (HD 2.3 b) definiert. Dann gilt (HD 2.7). Das ist alles unabhängig von (wirklichen, d.h. die Metrik einschränkenden) Feldgleichungen. Wenn Feldgleichungen hinzugenommen werden und $j_{2}=T_{\lambda}^{\mu} u_{\mu}$ gesetzt wird, kann eine explizite Darstellung für $t_{\lambda}$ berechnet werden, im Einsteinschen Fall ergibt sich (HD 2.5). Bei anderen Feldgleichungen (z. B. Jordanschen, oder bei spezieller Relativitätstheorie) ändert sich nur (HD 2.5), alles andere bleibt ungeändert.

4. Die von uns gewählte mathematische Präzisierung der Hönl-Dehnenschen Form des Machschen Prinzips [Text bei Gln. (23), (24)] wird in der Erwiderung als ,frei“" bezeichnet. Leider wird aber dort die richtige Formulierung auch nicht angegeben. Die auf S. 500 vor Formel (3.1) stehende Frage „Läßt sich..." ist nicht als richtige Formulierung zu verwenden, weil die Autoren nicht definieren, was die Einschränkung „bei gegebenem Bezugssystem $u_{\lambda}$ " mathematisch bedeuten soll; in der zu lösenden G1. (HD 3.2) kommt ja $u_{\lambda}$ gar nicht vor. Wenn die „Festhaltung des Bezugsystems“ bedeuten soll, daß für jede ,,mögliche“ Feldstärke $F_{\lambda \mu}$ zu gegebener Quelle $j_{\lambda}+t_{\lambda}$ gelten soll $F_{\lambda \mu}=u_{\mu \mid \lambda}-u_{\lambda \mid \mu}$, dann ist eben durch diese Gleichung $F_{\lambda \mu}$ bestimmt, und eine Eindeutigkeitsfrage kann gar nicht mehr gestellt werden. Wir haben deshalb die „Festhaltung des Bezugsystems" so aufgefaßt, daß die Gln. (23) und (24) bei gegebenem $u_{\lambda}$ bezüglich $\left(F_{\lambda \mu}, G_{\lambda \mu}\right)$, gelöst werden sollen, wobei natürlich $F_{\lambda \mu}=u_{\mu \mid \lambda}-u_{\lambda \mid \mu}$ nicht auch noch verlangt werden kann. Dann besteht, wie wir bewiesen haben, keine Eindeutigkeit z.B. beim Einstein-Kosmos. Wenn unsere Fassung nicht richtig sein soll, bleiben die Fragen: Was ist die strenge Fassung des Mach-Hönl-Dehnenschen Prinzips, und was ist darüber bewiesen? 